ИЗВЕСТИЯ АКАДЕМИИ НАУК ЭСТОНСКОИ ССР, ФИЗИКА - МАТЕМАТИКА PROCEEDINGS OF THE ACADEMY OF SCIENCES OF THE ESTONIAN SSR. PHYSICS * MATHEMATICS

$1988,37,4$

удК 621.382 .2

Б. ФРЕИДИН

\title{
ЭФФЕКТИВНЫЙ ЧИСЛЕННЫЙ АЛГОРИТМ МОДЕЛИРОВАНИЯ ЭЛЕКТРОТЕПЛОВЫХ ПРОЦЕССОВ В ОДНОМЕРНЫХ ПОЛУПРОВОДНИКОВЫХ СТРУКТУРАХ
}

\author{
(Представил Н. Алумяэ)
}

\section{1. Введение}

Теоретическое исследование электротепловых процессов в силовых полупроводниковых приборах (СПП) может быть выполнено с помощью эффективных численных моделей $\left[{ }^{1,2}\right]$, основанных на самосогласованном решении в одномерном приближении нелинейной системы дифференциальных уравнений полупроводника, содержащей уравнения непрерывности для дырок и электронов, уравнение Пуассона и уравнение полного тока, совместно с уравнением теплопроводности. Прогнозирование стойкости приборов к воздействию импульсной перегрузки током большой амплитуды сводится в этом случае к анализу температурных полей и вольт-амперных характеристик (BAX), рассчитанных с помощью этих моделей. Для корректного описания электротепловых процессов в структуре необходимо в первую очередь адекватно моделировать ВАХ в неизотермическом режиме с учетом таких нелинейных эффектов как, в частности, электронно-дырочное рассеяние (ЭДР) и Оже-рекомбинация.

Корректный учет влияния ЭДР на характеристики полупроводниковых структур в изотермическом приближении, заключающийся в изменении вида уравнений для плотностей токов и учета в них составляющих, описывающих взаимное увлечение носителей, рассмотрен в $[3,4]$. В настоящей работе учет ЭДР, развитый в $\left[{ }^{3}\right]$, обобщен на неизотермический режим.

Одним из наиболее эффективных подходов к решению нелинейной системы алгебраических уравнений, полученной после конечно-разностной аппроксимации исходной системы дифференциальных уравнений полупроводника, является одновременное совместное ее решение методом Ньютона $\left[{ }^{5}\right]$, что приводит к необходимости решения системы линейных алгебраических уравнений (СЛАУ) порядка $3 L$, где $L-$ количество узлов пространственной сетки.

Поскольку решение СЛАУ является основной операцией итерационного цикла при программной реализации алгоритмов, то при одинаковом количестве узлов сетки эффективность алгоритма можно повысить как за счет снижения порядка СЛАУ в результате сокращения числа исходных уравнений, так и за счет более эффективного метода решения СЛАУ.

Идея сокращения числа исходных уравнений для снижения порядка СЛАУ была впервые реализована в $\left[{ }^{6}\right]$, а затем использовалась в различных вариантах в $\left[{ }^{7,8}\right]$ при моделировании изотермических процессов в полупроводниковых структурах. 
В настоящей работе, являющейся непосредственным развитием $[2,8]$ предлагается эффективный численный алгоритм, предназначенный для моделирования неизотермических переходных процессов в СПП. Алгоритм основан на исключении напряженности электрического поля из системы трех конечно-разностных уравнений и сведении последней к двум уравнениям с концентрациями дырок и электронов в качестве переменных, в результате чего порядок СЛАУ снижается до $2 L$. Проводится сравнение эффективностей предлагаемого алгоритма с модифицированной версией алгоритма, изложенного в $\left[{ }^{2}\right]$, на примере расчета динамической неизотермической $\mathrm{BAX}$ силового кремниевого диода.

\section{2. Исходные уравнения и метод решения}

Система нелинейных дифференциальных уравнений, описывающая электротепловые процессы в полупроводниковой структуре, имеет в одномерном приближении следующий известный вид:

$$
\begin{aligned}
& \frac{\partial p}{\partial t}=-\frac{1}{q} \frac{\partial j_{p}}{\partial x}-R_{p}+G_{p}, \\
& \frac{\partial n}{\partial t}=\frac{1}{q} \frac{\partial j_{n}}{\partial x}-R_{n}+G_{n}, \\
& \frac{\partial E}{\partial t}=\frac{1}{\varepsilon \varepsilon_{0}}\left(j-j_{p}-j_{n}\right), \\
& \frac{\partial E}{\partial x}=\frac{q}{\varepsilon \varepsilon_{0}}(p-n+N), \\
& { }_{\mathrm{Q} C} C_{p} \frac{\partial T}{\partial t}=\frac{\partial}{\partial x}\left(\lambda \frac{\partial T}{\partial x}\right)+Q .
\end{aligned}
$$

В системе использованы стандартные обозначения, принятые в теории полупроводников. Здесь (1), (2) - уравнения непрерывности для дырок и электронов соответственно; (3) - уравнение плотности полного тока; (4) - уравнение Пуассона; (5) - уравнение теплопроводности.

Уравнения плотностей токов с учетом ЭДР, следующие из теории Авакьянца и др. $\left[{ }^{9}\right]$, рассмотренные в изотермическом случае также в $\left[{ }^{3}\right]$, имеют вид:

$$
j_{p}=\beta_{p p} j_{p 0}-\beta_{p n} j_{n 0} ; \quad j_{n}=\beta_{n n} j_{n 0}-\beta_{n p} j_{p 0} .
$$

Здесь $j_{p 0}, j_{n 0}-$ плотности токов, определяемые из традиционных уравнений плотностей дырочного и электронного токов без учета ӘДР:

$$
\begin{aligned}
& j_{p}=j_{p 0}=q \mu_{p} p E-q D_{p} \frac{\partial p}{\partial x}, \\
& j_{n}=j_{n 0}=q \mu_{n} n E+q D_{n} \frac{\partial n}{\partial x},
\end{aligned}
$$

где подвижности и коэффициенты диффузии связаны между собой соотношением Эйнштейна. Выражения для нелинейных коэффициентов $\beta$ приведены в $\left[{ }^{3}\right]$. В модели учитывались рекомбинации Шокли-РидаХолла и межзонная Оже-рекомбинация (все значения параметров и зависимости, использованные в модели, приведены в $\left.\left[{ }^{2}\right]\right)$.

Краевые условия для уравнений (1), (2) определялись из физи- 
ческой модели идеальных омических контактов на границе металлполупроводник, а краевые условия для уравнения теплопроводности приведены в $\left[{ }^{2}\right]$. В качестве начального условия для нестационарного теплового процесса была принята одинаковая температура вдоль всей структуры.

Временная зависимость плотности полного тока $j(t)$, имеющая в общем случае произвольный характер, считалась заданной. В результате решения уравнений (1)-(7) наряду с распределениями искомых переменных вдоль структуры путем интегрирования напряженности электрического поля отыскивается величина напряжения, приложенного к контактам структуры, и тем самым определяется BAX.

Исходная нелинейная система дифференциальных уравнений решается методом конечных разностей. Модель и метод решения изложены в $\left[{ }^{2}\right]$. После конечно-разностной аппроксимации и линеаризации исходной системы уравнений полупроводника решается СЛАУ с количеством уравнений $3(L-2)+1$. Уравнение теплопроводности решается отдельно.

Слабозаполненные матрицы коэффициентов имеют 7-диагональную ленточную структуру в случае, если независимыми переменными являются $p, n, E$ и ӘДР учитывается традиционно путем суммирования обратных величин подвижностей. Корректный учет ЭДР увеличивает количество диагоналей до 9 из-за появления перекрестных связей в уравнениях плотностей токов. Алгоритм, основанный на одновременном решении СЛАУ в переменных $p, n, E$ с 9-диагональной матрицей коэффициентов, будем считать в дальнейшем исходным для сравнения эффективностей алгоритмов и назовем 9 Д.

С целью снижения порядка СЛАУ за счет сокращения количества уравнений, напряженность электрического поля исключается из конечно-разностного уравнения полного тока и подставляется в систему двух конечно-разностных уравнений непрерывности. После выполнения преобразований, аналогичных $\left[{ }^{8}\right]$, получается СЛАУ с 7 -диагональной матрицей коэффициентов и количеством уравнений $2(L-2)$. Отметим, что при традиционном учете ЭДР матрица содержит 6 диагоналей. Численный алгоритм, основанный на исключении напряженности электрического поля и одновременном решении преобразованной системы с переменным $p, n$, назовем 7 Д.

Решение СЛАУ в обоих алгоритмах проводилось методом исключения Гаусса с помощью стандартной программы DGELB $\left[{ }^{10}\right]$, входящей в состав программного обеспечения ОС ЕС ЭВМ. Помимо этого в алгоритме 7 Дл для решения СЛАУ использовался матрично-векторный аппарат, развитый в $\left[{ }^{11}\right]$ и реализованный в виде специальной подпрограммы.

\section{3. Результаты расчета}

Эффективность численных алгоритмов исследовалась путем расчета динамической неизотермической ВАХ кремниевого силового диода при воздействии мощного синусоидального импульса прямого тока перегрузки амплитудой $2,0 \mathrm{kA} / \mathrm{cm}^{2}$ и длительностью $10 \mathrm{mc}$. До воздействия импульса тока структура находилась при температуре $300 \mathrm{~K}$ и через нее протекал практически негреющий прямой ток плотностью $10 \mathrm{~A} /$ см$^{2}$. При расчетах использовалась следующая структура выпрямительного элемента: 1 - медь (толщина 10 мм); $2-$ серебро (100 мкм); 3 - вольфрам $(2,2$ мм); 4 - силумин (100 мкм); 5 $p^{+}-n-n^{+}$кремниевая диодная структура с диффузионным распределением примеси (510 мкм); 6 - алюминий (15 мкм); 7 - молибден 
(500 мкм); 8 - серебро (100 мкм); 9 - медь (10 мм). Тепловые контактные сопротивления между слоями структуры были приняты одинаковыми и равными $0,01 \mathrm{Kcm}^{2} /$ Вт. При расчетах использовалось экспериментально снятое распределение концентрации примеси вдоль полупроводниковой структуры. Параметры рекомбинационной модели Шокли-Рида-Холла были приняты равными 0,2 мкс в эмиттерах и 5 мкс в базе структуры.

Все расчеты проводились на ЭВМ ЕС 1055 M с помощью программы ДИНАМИТ-2, предназначенной для моделирования электротепловых процессов в СПП и реализованной на основе модели $\left[{ }^{2}\right]$. Изложенные алгоритмы реализованы в виде программных модулей, входящих в состав программы.

BAX структуры рассчитывались при следующих условиях: количество узлов неравномерной сетки было принято 143 (из них 60 в полупроводнике); равномерный временной шаг при расчете электротепловых процессов до 10 мс составлял 0,5 мс; критерием сходимости итерационного процесса служило максимальное значение модуля приращения потенциала, не превышающее 2,6 мкВ (что соответствует нормализованному значению $10^{-4}$ ).

Численные эксперименты показали, что оба алгоритма обладают совершенно одинаковой зависимостью сходимости (количества итераций) от величины плотности протекающего тока (или напряжения, приложенного к структуре). Аналогичная зависимость приведена в $\left[{ }^{5}\right]$ и характеризуется лишь незначительным изменением числа итераций в широком диапазоне изменения плотностей токов и напряжений.

Сравнение предложенных алгоритмов с ранее известными показывает, что в исследуемых алгоритмах не наблюдалось ухудшения сходимости с ростом плотности тока, обусловленной взаимосвязью решаемых уравнений, как сообщалось в [ $\left.{ }^{4}\right]$. Число итераций в расчетах при вышеуказанных условиях находилось в пределах $10-13$ на каждом временном шаге. Указанное обстоятельство объясняется преимуществом методов совместного решения уравнений $\left[{ }^{5}\right]$ по сравнению с методом попеременного решения $\left[{ }^{4}\right]$, которому в существенной степени присущи недостатки классического метода Гуммеля $\left[{ }^{12}\right]$, а именно, низкая сходимость при высоком уровне инжекции.

Расчеты показали, что при одинаковой сходимости обоих исследуемых алгоритмов общий выигрыш времени счета алгоритма 7 Д по сравнению с 9Д составляет приблизительно 1,3 раза и достигается за счет следующих двух факторов: в результате снижения порядка СЛАУ, приводящего к сокращению времени счета при одном и том же методе, а также использования более эффективного метода решения СЛАУ.

Типичное время счета переходных электротепловых процессов в структуре составляет при вышеуказанных условиях в среднем 5 минут при одном значении амплитуды импульса тока.

Разработанный эффективный алгоритм позволяет сократить время счета программы ДИНАМИТ-2 в 1,3 раза и может быть использован в программном обеспечении САПР СПП для расчета неизотермических характеристик и прогнозирования ударного тока СПП.

Автор выражает благодарность Э. Велмре за полезное обсуждение рукописи и И. Нурсте за помощь при выполнении расчетов. 
1. Adler, M. S., Glascock, H. H. IEEE Trans. Electron Dev., 26, № 7, 1085-1091 (1979).

2. Велмре Э. Э., Фрейдин Б. П. Электронное моделирование, 5, № 1, 73-76 (1983).

3. Велмре Э. Э., Пироженко А. А., Удал А. Э. Электронное моделирование, 7, № 4, $66-71$ (1985).

4. Мнацаканов T. Т., Ростовцев И. Л., Филатов Н. И. Электронное моделирование, 8, № $1,40-43$ (1986).

5. Велмре Э. Э., Удал А. Э., Фрейдин Б. П. Электронное моделирование, 3 , № 4, $85-88$ (1981).

6. Hachtel, G. D., Joy, R. C., Cooley, J. W. Proc. IEEE, 60, № 1, 86-98 (1972).

7. Мулярчик С. П., Абрамов Н. Н., Соловьев В. Г. Изв. вузов. Радиоэлектроника, 23, № 6, 55-60 (1980).

8. Фрейдин Б. П. Тр. Таллинск. политех. нн-та, вып. 538, 39-48 (1982).

9. Авакьянц Г. М., Мурысин В. Н., Сандлер Л. С., Тешиабаев А., Юровский А. В. Радиотехника и электроника, 8, № 11, 1919-1926 (1963).

10. Матричная алгебра и линейная алгебра (Сб. научных программ на ФОРТРАНе). М., «Статистика», 1974.

11. Kurata, M. IEEE Trans. Electron Dev., 19, № 11, 1207-1215 (1972).

12. Gummel, H. K. IEEE Trans. Electron Dev., 11, № 10, 455-465 (1964).
Институт термофизики и электрофизики Академии наук Эстонской ССР
Поступила в редакцию 26/VI 1987

\section{B. FREIDIN}

\section{EFEKTIIVNE NUMBRILINE ALGORITM ELEKTROTERMILISTE PROTSESSIDE MODELLEERIMISEKS OHEMOOTTMELISTES POOLJUHTSTRUKTUURIDES}

On kirjeldatud efektiivset numbrilist algoritmi elektrotermiliste üleminekuprotsesside modelleerimiseks, mis pōhineb pooljuhtide fundamentaalvõrrandite süsteemi ja soojusjuhtivuse võrrandi lahendamisel. Elektronaugu hajumise korrektseks arvestamiseks on voolutiheduste võrrandites arvesse võetud laengukandjate omavahelist kaasahaaramist kirjeldavad liikmed. Algoritm pōhineb muutujate arvu vähendamisel kolme diferentsvōrrandiga süsteemist elektrivälja väljaarvamisega ja süsteemi teisendamisel kaheks võrrandiks, milles muutujad on aukude ja elektronide kontsentratsioonid. Võrreldes varem kasutatud algoritmiga vähendab kõnesolev algoritm arvutusaega 1,3 korda.

\section{B. FREIDIN}

\section{AN EFFICIENT NUMERICAL ALGORITHM FOR MODELING ELECTROTHERMAL PROCESSES IN ONE-DIMENSIONAL SEMICONDUCTOR STRUCTURES}

An efficient numerical algorithm for modeling the electrothermal transient processes in semiconductor structures based on the self-consistent solution of the fundamental set of semiconductor equations and a heat-flow equation is described.

In order to take into account the electron-hole scattering correctly, the additional terms describing drag effect of carriers in current equations are considered.

An increase in the efficiency of the proposed algorithm is obtained by the reduction of the number of variables due to the electric field elimination in the set of initial equations. As it was shown by the comparison of the present and the previously published algorithms, the application of the developed algorithm will reduce the computing time by the factor of 1.3 . 